\title{
Negative refraction in natural ferromagnetic metals
}

\author{
S. Engelbrecht, ${ }^{1}$ A. M. Shuvaev, ${ }^{1}$ Y. Luo, ${ }^{2}$ V. Moshnyaga,${ }^{2}$ and A. Pimenov ${ }^{1}$ \\ ${ }^{1}$ Experimentelle Physik IV, Universität Würzburg, 97074 Würzburg, Germany \\ ${ }^{2}$ I. Physikalisches Institut, Universität Göttingen, 37073 Göttingen, Germany
}

(Dated: September 4, 2018)

\begin{abstract}
It is generally believed that Veselago's criterion for negative refraction cannot be fulfilled in natural materials. However, considering imaginary parts of the permittivity $(\varepsilon)$ and permeability $(\mu)$ and for metals at not too high frequencies the general condition for negative refraction becomes extremely simple: $\operatorname{Re}(\mu)<0 \Rightarrow \operatorname{Re}(n)<0$. Here we demonstrate experimentally that in such natural metals as pure Co and FeCo alloy the negative values of the refractive index are achieved close to the frequency of the ferromagnetic resonance. Large values of the negative refraction can be obtained at room temperature and they can easily be tuned in moderate magnetic fields.
\end{abstract}


The topic of negative refraction [1] has attracted much research interest in the last years [2, 3]. Various possible realizations of a negative index material has been proposed [4-10]. Experimental demonstrations of negative refraction in metamaterials [11, 12] and multilayers [7] are based on the classical criterion by Veselago which requires simultaneous negativity of electric permittivity $\varepsilon$ and magnetic permeability $\mu$ [1]. However, natural materials with these properties most probably do not exist. An alternative way to achieve negative refraction in natural material may try to use the extended criterion [13, 14] which takes into account imaginary parts of the permittivity and permeability:

$$
\left(\varepsilon_{1}+\left|\varepsilon^{*}\right|\right)\left(\mu_{1}+\left|\mu^{*}\right|\right)<\varepsilon_{2} \mu_{2}
$$

This equation is straightforwardly derived from the inequality $\operatorname{Re}\left(\sqrt{\varepsilon^{*} \mu^{*}}\right)<0$. Here $\varepsilon^{*}=\varepsilon_{1}+i \varepsilon_{2}$ denotes the complex dielectric permittivity and $\mu^{*}=\mu_{1}+i \mu_{2}$ the complex magnetic permeability, respectively. Eq. (1) has an interesting consequence if we consider the electrodynamics of metals [15]. For metals at frequencies far below the scattering rate the imaginary part of the permittivity dominates: $\varepsilon_{1}<<\varepsilon_{2}$ and, therefore, $\left|\varepsilon^{*}\right| \approx \varepsilon_{2}$. In this case Eq. (11) can be transformed to a simple final condition for the negative refraction: $\mu_{1}<0 \Rightarrow \operatorname{Re}\left(n^{*}\right)<0$. This condition should be fulfilled in ferromagnetic metals close to the ferromagnetic resonance if the strength of the mode is high enough. First experimental demonstration of negative refraction in a ferromagnetic metal [9] utilized the ferromagnetic resonance in a $\mathrm{Ca}$ doped $\mathrm{LaMnO}_{3}$. Although a sufficiency of the simple condition $\mu_{1}<0$ for metals has been proven, the existence of natural materials with negative refraction still had to be demonstrated.

In this Letter we present the results of the refractive index experiments in real natural metals. As examples of natural ferromagnetic metals we have chosen pure Cobalt and $\mathrm{Fe} / \mathrm{Co}$ alloy. Using polarization and phase controlled experiments in the millimeter wave range we demonstrate that close to the frequency of the ferromagnetic resonance the refractive index of Co and FeCo alloy indeed goes deep into the negative regime.

Pure Cobalt and $\mathrm{Fe}_{0.5} \mathrm{Co}_{0.5}$ alloy were prepared as polycrystalline thin films by magnetron sputtering technique. The thickness of both samples was $150 \pm 30 \mathrm{~nm}$. As substrate $\mathrm{MgO}$ was used, whose millimeter wave properties have been determined in a separate experiment as $\mathrm{n}_{M g O}=(3.09 \pm 0.01)+1.4 \cdot 10^{-9}(T[K])^{3}$. Here the temperature $T$ is given in Kelvin. The imaginary part of the refractive index of the $\mathrm{MgO}$ substrate in the frequency range of the 


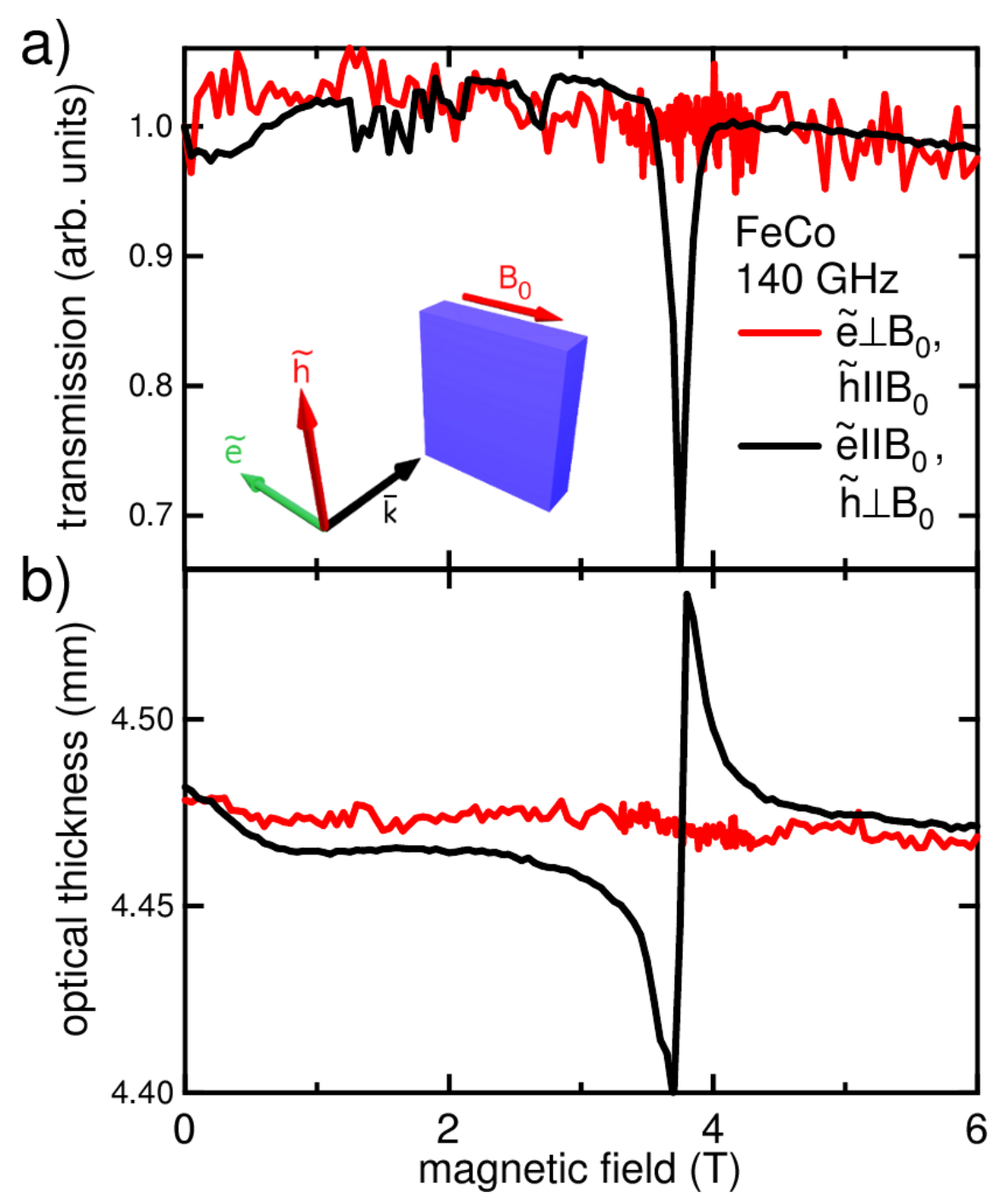

FIG. 1: a) transmittance and b) phase shift of FeCo alloy for two different excitation geometries at $\mathrm{T}=170 \mathrm{~K}$. Within the geometry $\tilde{h} \perp B_{0}$ the ferromagnetic resonance can be excited, whereas for $\tilde{h} \| B_{0}$ no excitation occurs. The inset sketches the magnetically active geometry $\left(\tilde{h} \perp B_{0}\right)$. Here $\bar{k}$ denotes the wave vector, $\tilde{h}$ and $\tilde{e}$ are the ac magnetic and electric fields of the radiation, and $\mathrm{B}_{0}$ is the static external magnetic field.

present experiment was below $1 \cdot 10^{-4}$ at all temperatures.

The experiments in the millimeter frequency range were carried out in a Mach-Zehnder interferometer setup [16, 17] using backward wave oscillators as radiation source. This spectrometer enables to measure both the transmittance and phase shift as function of frequency, temperature or external applied magnetic field within controlled polarization geometries. Magnetic field experiments were carried out using a split coil magnet with 
polypropylene windows.

The experimental spectra obtained were analyzed using the Fresnel formulas for the complex transmission coefficient of the substrate-film system [16-18]. A non-trivial problem of the experiment is the separation of dielectric and magnetic properties of the sample. In this case four independent experimental values are necessary. In present experiments, we obtained the transmittance and phase shift of the sample within two different polarizations of the incident radiation, $\tilde{h} \| B_{0}$ and $\tilde{h} \perp B_{0}$. Here $\tilde{h}$ is the ac magnetic field of the radiation and $B_{0}$ is the external magnetic field. From the solution of the Bloch's equations for the magnetic moments in external magnetic fields it follows that nonzero magnetic susceptibility can only be obtained in the geometry $\tilde{h} \perp B_{0}$ [19]. The inset of Fig. 1] shows the geometry in which the ferromagnetic resonance is excited. To determine the parameters of the ferromagnetic resonance a Lorentz line shape was used, i.e. the magnetic permeability was taken as

$$
\mu^{*}(B)=1+\frac{\Delta \mu B B_{0}}{B^{2}-B_{0}^{2}-i B_{0} \Gamma} .
$$

Here $\Gamma$ is the width of the resonance, $B_{0}$ the resonance field and $\mathrm{B}$ the external applied magnetic field.

Fig. 1 shows the transmittance and phase shift of the FeCo alloy at $\mathrm{T}=170 \mathrm{~K}$ and at a frequency of $140 \mathrm{GHz}$ in both excitation geometries. As can be clearly seen in the geometry $\tilde{h} \| B_{0}$ no excitation of the ferromagnetic resonance occurs $\left(\mu^{*}=1\right)$ and, therefore, we can use this geometry to determine the dielectric properties of the metal films. These properties are obtained from the absolute transmittance spectra in zero field and are well described by conventional Drude conductivity of a metal in the millimeter range (Table I).

Furthermore, in the nonmagnetic geometry no magnetoresistance occurs within our experimental resolution neither in the FeCo alloy nor in pure Co. Therefore we can assume a magnetic field independent behavior of the permittivity. In contrast, in the geometry $\tilde{h} \perp B$ the resonance can be excited which allows to independently determine the permeability. The excitation frequency is dependent on the resonance field and follows the relationship $\omega_{\text {res }}=\gamma \sqrt{\left(H_{0}+4 \pi M_{0}\right) H_{0}}$ due to the geometry of the experiment (Voigt geometry) [20]. Here $\omega_{\text {res }}$ denotes the excitation frequency, $\gamma$ is the gyromagnetic ratio and $M_{0}$ is the static magnetization. Using the dielectric permittivity obtained from the magnetically inactive geometry we are able to calculate the magnetic permeability which is shown in Fig. 2 for the Co sample at $\mathrm{T}=190 \mathrm{~K}$. 


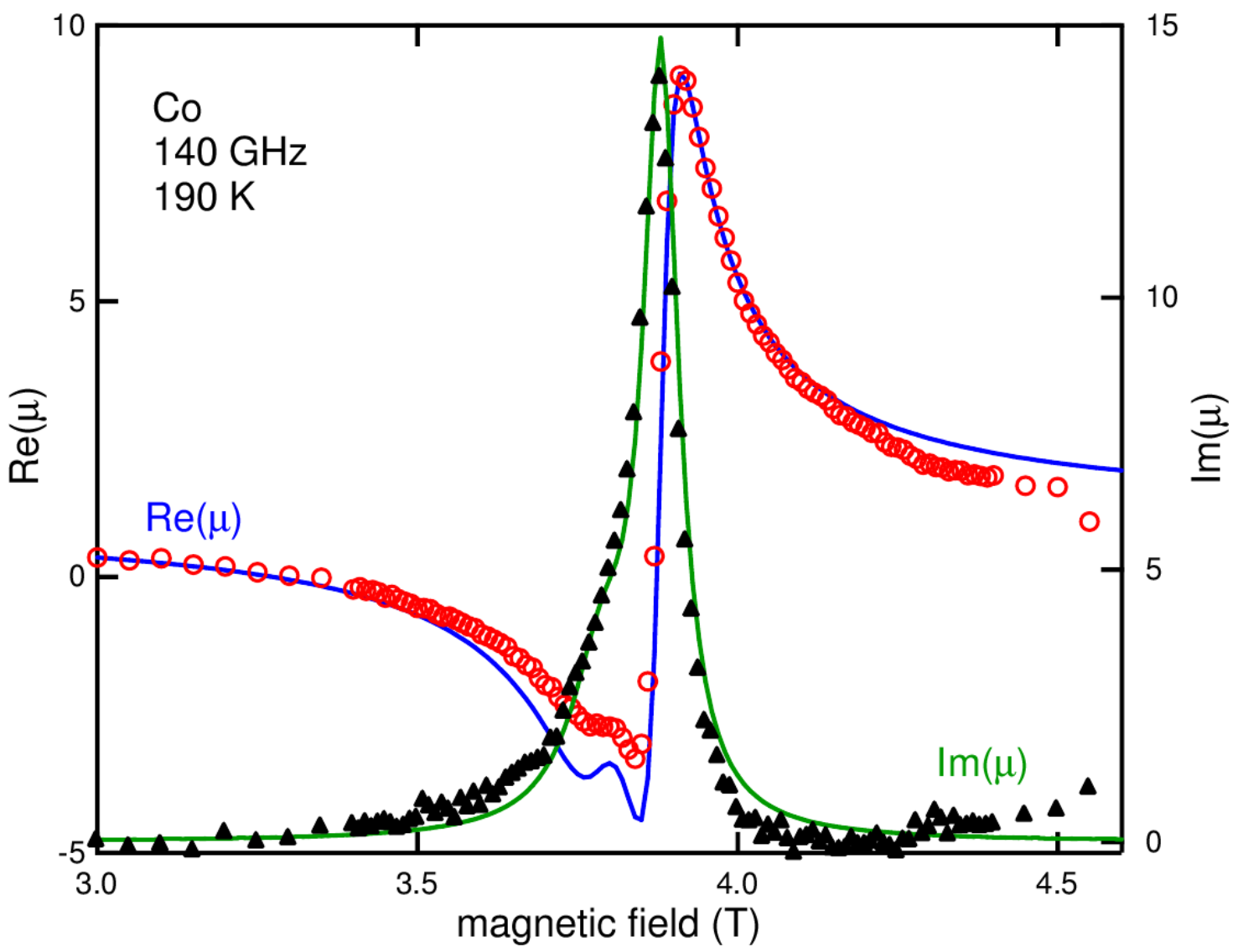

FIG. 2: Complex magnetic permeability obtained from transmission and phase shift data (symbols) and fitted using Eq. 22(solid lines). Close to the resonance the real part of the permeability becomes negative.

Table I summarizes the film properties for FeCo and Co at two different excitation frequencies. For both samples we get quite similar electrodynamic parameters. Weak temperature dependence of the resonance fields is due to the decreasing static magnetization with increasing temperature. These effects are quite small, since the Curie temperatures of both Co and FeCo are far above room temperature (Co: $\mathrm{T}_{C}=1390 \mathrm{~K}$ [21], FeCo: $\mathrm{T}_{C}=1253 \mathrm{~K}$ [22]).

With the dielectric permittivity and the magnetic permeability determined, we can now calculate the refractive index by $\mathrm{n}=\sqrt{\varepsilon^{*} \mu^{*}}$. Here we note that due to a continuous variation of the refractive index as function of external magnetic fields the sign of the square root is obtained automatically on crossing the imaginary axis. Fig. 3 a) and b) show the magnetic field dependence of the complex refractive index of the FeCo and Co sample, respectively. The shaded area denotes the range in which the refractive index becomes negative. Here we 
TABLE I: Electrodynamic parameters for FeCo and Co samples at two different excitation frequencies and temperatures.

\begin{tabular}{ccccc}
\hline \hline sample & \multicolumn{2}{c}{ FeCo } & \multicolumn{2}{c}{ Co } \\
frequency $[\mathrm{GHz}]$ & 140 & 182 & 140 & 180 \\
\hline $\mathrm{T}=10 \mathrm{~K}$ & & & & \\
$\mathrm{~B}_{0}[\mathrm{~T}]$ & $3.73 \pm 0.01$ & $5.12 \pm 0.01$ & $3.87 \pm 0.01$ & $5.20 \pm 0.01$ \\
$\Delta \mu$ & $0.32 \pm 0.05$ & $0.16 \pm 0.05$ & $0.20 \pm 0.05$ & $0.13 \pm 0.05$ \\
$\Gamma[\mathrm{T}]$ & $0.07 \pm 0.02$ & $0.08 \pm 0.02$ & $0.08 \pm 0.02$ & $0.11 \pm 0.02$ \\
$\sigma\left[10^{6} \Omega^{-1} m^{-1}\right]$ & $8 \pm 2$ & $12 \pm 2$ \\
\hline $\mathrm{T}=300 \mathrm{~K}$ & & & \\
$\mathrm{~B}_{0}[\mathrm{~T}]$ & $3.76 \pm 0.01$ & $5.17 \pm 0.01$ & $3.89 \pm 0.01$ & $5.21 \pm 0.01$ \\
$\Delta \mu$ & $0.29 \pm 0.05$ & $0.27 \pm 0.05$ & $0.38 \pm 0.05$ & $0.21 \pm 0.05$ \\
$\Gamma[\mathrm{T}]$ & $0.08 \pm 0.02$ & $0.1 \pm 0.02$ & $0.07 \pm 0.02$ & $0.11 \pm 0.02$ \\
$\sigma\left[10^{6} \Omega^{-1} m^{-1}\right]$ & $6 \pm 1$ & $6 \pm 1$ \\
\hline \hline
\end{tabular}

see that even at room temperature these metals show a negative index of refraction, which is also highly tunable by the applied magnetic field. We note that the second weak resonance which can be seen in these plots (at $\sim 3.7 \mathrm{~T}$ for FeCo and $\sim 3.8 \mathrm{~T}$ for $\mathrm{Co}$ ) most probably arises due to inhomogeneities in the film magnetization [23]. The estimate of the best value of the figure of merit for our samples gives $|n| / \kappa \sim 0.8$. This value is already close to the theoretical limit $|n| / \kappa=1$ for ferromagnetic metals.

A remaining problem with the present materials is the high absorption coefficient in the region of negative refraction. High absorption values leads to small transmissions of the order of $10^{-4}$ and have to be overcome for possible applications. A possible solution to this problem could be the usage of either superconductors or very clean metals. In these cases and in the millimeter range the conductivity will be purely imaginary, i.e. no internal absorption will be present. Unfortunately, at current stage of research both solution would be difficult, as e.g. magnetism and superconductivity tend to exclude each other.

In conclusion, we have shown that the refractive index in natural ferromagnetic metals becomes negative close to the frequency of the ferromagnetic resonance. We have investi- 


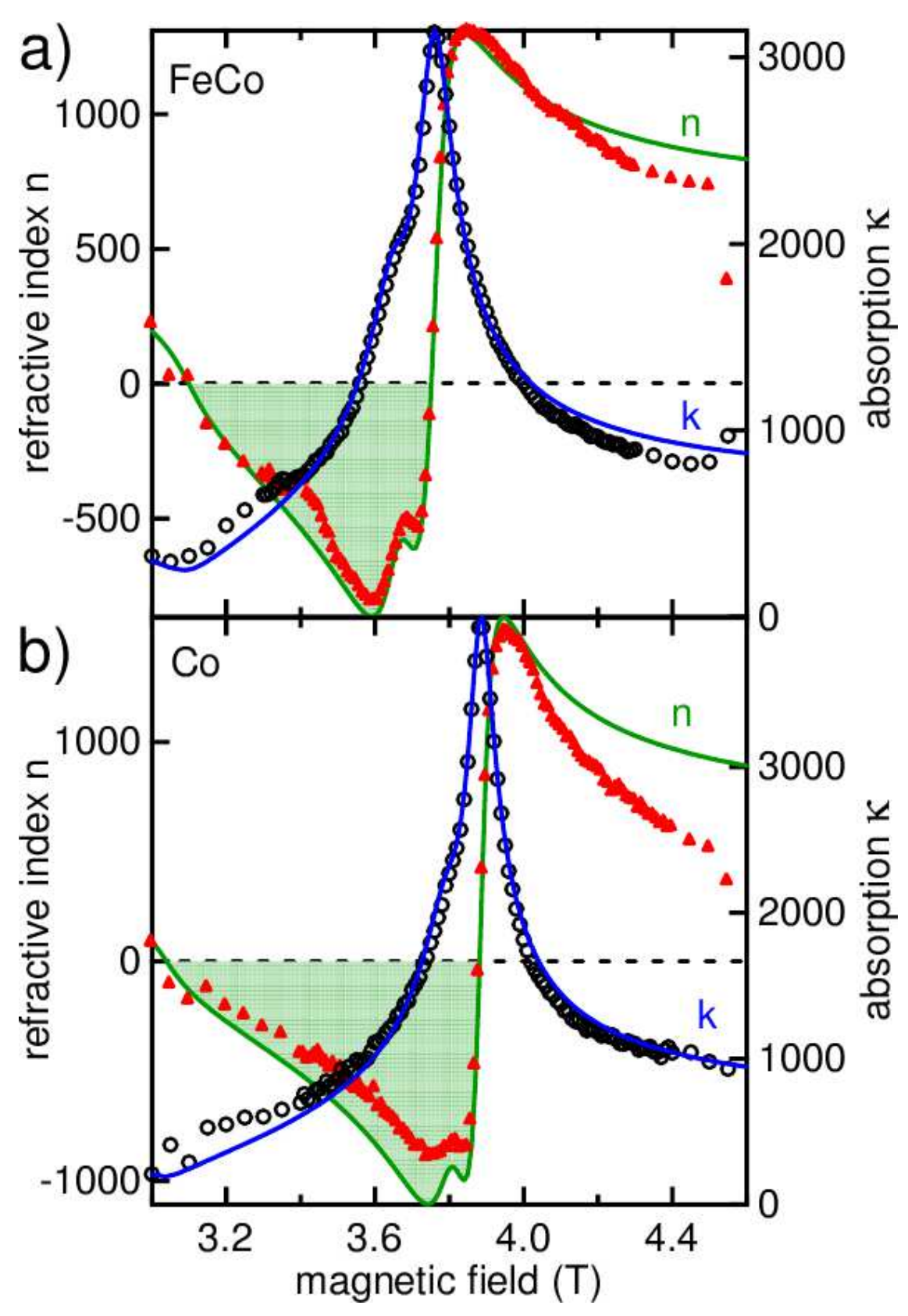

FIG. 3: Magnetic field dependent refractive index for the a) FeCo alloy and b) metallic Co at $\mathrm{T}=300 \mathrm{~K}$ and at $140 \mathrm{GHz}$. The shaded area denotes the range in which the real part of the refractive index becomes negative. Symbols - experimental data, solid lines are fits using Eq. (2)

gated pure Co and FeCo alloy both revealing negative values of the refractive index over a large temperature range and at different excitation frequencies. Both metals show large negative index of refraction at room temperature which is highly tunable by the external magnetic field. 
[1] V. G. Veselago, Sov. Phys. Usp. 10, 509 (1968).

[2] J. B. Pendry and D. R. Smith, Phys. Today 57, 37 (2004).

[3] D. R. Smith, J. B. Pendry, and M. C. K. Wiltshire, Science 305, 788 (2004).

[4] D. R. Smith, W. J. Padilla, D. C. Vier, S. C. Nemat-Nasser, and S. Schultz, Phys. Rev. Lett. 84, $4184(2000)$.

[5] E. Cubukcu, K. Aydin, E. Ozbay, S. Foteinopoulou, and C. M. Soukoulis, Nature 423, 604 (2003).

[6] J. B. Pendry, Science 306, 1353 (2004).

[7] A. Pimenov, A. Loidl, P. Przyslupski, and B. Dabrowski, Phys. Rev. Lett. 95, 247009 (2005).

[8] V. A. Podolskiy and E. E. Narimanov, Phys. Rev. B 71, 201101 (2005).

[9] A. Pimenov, A. Loidl, K. Gehrke, V. Moshnyaga, and K. Samwer, Phys. Rev. Lett. 98, 197401 (2007).

[10] J. Valentine, S. Zhang, T. Zentgraf, E. Ulin-Avila, D. A. Genov, G. Bartal, and X. Zhang, Nature 455, 376 (2008).

[11] R. A. Shelby, D. R. Smith, and S. Schultz, Science 292, 77 (2001).

[12] W. J. Padilla, D. N. Basov, and D. R. Smith, Mater Today 9, 28 (2006).

[13] M. W. McCall, A. Lakhtakia, and W. S. Weiglhofer, Eur. J. Phys. 23, 353 (2002).

[14] A. D. Boardman, N. King, and L. Velasco, Electromagnetics 25, 365 (2005).

[15] M. Dressel and G. Grüner, Electrodynamics of Solids: Optical Properties of Electrons in Matter (Cambridge University Press, Cambridge, 2002), 1st ed.

[16] A. Pimenov, S. Tachos, T. Rudolf, A. Loidl, D. Schrupp, M. Sing, and R. Claessen, Physical Review B 72, 035131 (2005).

[17] G. V. Kozlov and A. A. Volkov, in Millimeter and Submillimeter Wave Spectroscopy of Solids, edited by G. Grüner (Springer, Berlin, 1998), p. 51.

[18] M. Born and E. Wolf, Principles of Optics (Cambridge University Press, 2006).

[19] A. Abragam and B. Bleaney, Electron Paramagnetic Resonance of Transition Ions (Dover, New York, 1970).

[20] A. K. Zvezdin and V. A. Kotov, Modern Magnetooptics and Magnetooptical Materials (Institute of Physics Publ., Bristol, 1997). 
[21] P. Mohn and E. P. Wohlfarth, J. Phys. F: Met. Phys. 17, 2421 (1987).

[22] A. M. James and M. P. Lord, Macmillan's Chemical and Physical Data (The Macmillan Press Ltd, 1992).

[23] A. V. Semeno, V. V. Glushkov, A. V. Bogach, N. E. Sluchanko, A. V. Dukhnenko, V. B. Fillippov, N. Y. Shitsevalova, and S. V. Demishev, Physical Review B 79, 014423 (2009). 